\title{
A New Approach: About the Appearance of "Dark Matter" Effects in the Process of Expansion of the Universe*
}

\author{
Leonid Sitnikov \\ L. S. Sitnikov New York City Transit Authority, New York, USA \\ Email: sitnikov@cs.com
}

How to cite this paper: Sitnikov, L. (2021) A New Approach: About the Appearance of "Dark Matter" Effects in the Process of Expansion of the Universe. Journal of Modern Physics, 12, 1040-1047.

https://doi.org/10.4236/jmp.2021.127065

Received: April 19, 2021

Accepted: May 25, 2021

Published: May 28, 2021

Copyright $\odot 2021$ by author(s) and Scientific Research Publishing Inc. This work is licensed under the Creative Commons Attribution International License (CC BY 4.0).

http://creativecommons.org/licenses/by/4.0/

\section{(c) (i) Open Access}

\begin{abstract}
The paper considers cosmological objects belonging to fundamentally different classes that do not intersect with each other. Firstly, these are objects that make up a pure Hubble stream. Secondly, these are objects that have constant proper distances. These include planets, stars, and galaxies in gravitationally coupled systems. They all do not participate in the Hubble stream as independent objects. It is shown that the commoving reference system and proper reference system standardly used in cosmology change places with each other when switching from considering Hubble objects to "planets". The features of the evolution (more precisely, devolution, degradation, reverse development) of the latter were analyzed and it was found that the cosmological acceleration of all "planets", in contrast to Hubble objects, coincides in order of magnitude with the fundamental value of $H^{2} R$. As applied to the Pioneers anomaly, this approach allowed us to obtain the calculated value of cosmological acceleration, which coincides in order of magnitude with the observed value. It seems that this approach is applicable also to other local gravitationally coupled systems and allows us to explain the characteristic flattened shape of the orbital curves of stars and galaxies by the fact that the influence of the fundamental cosmological acceleration $H^{2} R$ is added to the system's own gravitational field.
\end{abstract}

\section{Keywords}

Dark Matter, Expansion of the Universe, Anomalous Pioneer Effect

\section{Introduction}

The expansion of the universe is described by general relativity. General relativi-

${ }^{\star}$ In dedicaton to Olga Kreydik. 
ty in combination with the cosmological principle unambiguously leads to Friedmann's cosmological models, i.e. to space-time with the Friedman-Robertson-Walker (FRU) metric:

$$
D s^{2}=-c^{2} d t^{2}+R^{2}(t)\left\{d \chi^{2}+S k^{2}(\chi)\left[d \theta^{2}+\sin ^{2} \theta d \Phi^{2}\right]\right\}
$$

Here $\chi$ is the commoving coordinate, $R(t)$ (sometimes $R$ ) is a scale factor with the dimension of distance, increasing with time in the expanding universe, and $S k(\chi)=(\sin \chi, \chi$ or $\operatorname{sh} \chi)$, where $\chi$ depends on the used model of the universe (closed, flat, open). Along with the factor $R(t)$ and the commoving coordinate $\chi$, the scale factor $R_{0}$, which does not depend on $t$, and the dimensionless scale factor $a=R\left(t_{1}\right) / R\left(t_{2}\right)$ are often used.

The proper distance $D$ from the origin to the location of interested star (galaxy) is the product of the scale factor $R(t)$ (its value is usually taken equal to the size of the universe at the present time) by the commoving coordinate:

$$
D=R(t) \chi
$$

Obviously, $\chi=D / R(t)$ coincides with $a=R\left(t_{1}\right) / R\left(t_{2}\right)$ for $D=R\left(t_{1}\right)$ and $R(t)=$ $R\left(t_{2}\right)$. The overall speed of a star, including escape velocity and peculiar velocity, is:

$$
D^{\prime}=R(t)^{\prime} \chi+R(t) \chi^{\prime}=v_{\text {reg }}+v_{p e c}
$$

Since in what follows we will be interested in the forces and accelerations arising in the process of the expansion of the universe, we will also write down the second derivative of the distance (further, instead of $R(t)$, we will use $R$ ):

$$
D^{\prime \prime}=\left(R^{\prime \prime} \chi+R^{\prime} \chi^{\prime}\right)+\left(R^{\prime} \chi^{\prime}+R \chi^{\prime \prime}\right)=\left(v_{\text {reg }}\right)^{\prime}+\left(v_{\text {pec }}\right)^{\prime}
$$

The commoving frame of reference (hereinafter referred to as FR) must exactly accompany the selected objects (in cosmology participants in the Hubble flow), must be glued to them so that, as a result, for any proper motions, these objects remain motionless in such a FR. Therefore, in the commoving, or Lagrangian [1] FR $t-\chi$ (often more convenient $t-R_{0} \chi$ ), the world lines of galaxies, strictly obeying the Hubble law, are vertical straight lines $\chi=\chi_{i}$. The deviation of any world line from the vertical, indicates that the corresponding object has dropped out of the purely Hubble flow, i.e. about the presence of his peculiar speed.

A different picture is observed in proper, or Euler's [1] FR $t-D$, which is much more familiar to us. Here, the same Hubble objects with $\chi=\chi_{i}$ represent a bundle of world lines $D=R(t) \chi_{i}$, diverging from the origin. Here there is a dependence on time, and it is clear that the universe is expanding. The connection between the two cases is given by the law of motion (1), which expresses the proper, or Euler, coordinates $D$ through the commoving, or Lagrangian, $\chi_{i}$ and time.

In a contracting universe, expression (1) for the proper distance will change to

$$
D=\left(R_{0}-R\right) \chi
$$

leading to the appropriate transformation of the expressions for speed and acceleration. 


\section{Analysis of Cosmological Accelerations}

And now let us represent in the same FR $t-D$ and FR $t-\chi$ the world lines of cosmological objects that do not take sovereign participation in the global expansion of the universe. These include objects that are part of local gravitationally coupled systems, such as the planets of the Solar System or galaxies that rotate in circular orbits in a cluster of galaxies and, therefore, have fixed proper distances $D_{i}$ from the center of rotation. We again see the world lines in the form of vertical lines. But now these are world lines $D=D_{i}$ of "non-Hobbles" (planet-like objects) in FR $t-D$, but not world lines $\chi=\chi_{i}$ of Hubble objects in FR $t$ $-\chi$, which are usually dealt with in cosmology. The verticality of the world lines is indisputable evidence that they are depicted in the commoving FR. Thus, in the case of vertical world lines, we are dealing with two fundamentally different classes of cosmological objects. First, these are objects with world lines $\chi=\chi_{i}$, which are full-fledged participants in the Hubble flow, forming the Hubble flow; and, secondly, these are objects with $D=D_{i}$ (it will be more convenient to use $D_{i}$ instead of $R_{0}$ to denote time-independent distances in FR $t-D$ that do not independently participate in this flow. These classes are associated with different commoving FR. And not just different, but in a sense diametrically opposite, mutually complementary.

We emphasize again: for any objects with $D=D_{i}$, the commoving FR is by no means the FR $t-\chi$, as for Hubble objects, but the FR $t-D$ instead. Naturally, when moving from considering Hubble objects to analyzing the motion of local objects, i.e. when passing from FR $t-\chi$ to FR $t-D$ the Lagrangian and Euler descriptions trade places, and therefore now the law of motion is not $D=R(t) \chi_{i}$, as in (1) for Hubble objects, but vice versa:

$$
\chi=D_{i} / R(t)
$$

As it follows from (4), the proper distance $\chi$ for planet-like objects necessarily decreases with time $t$ (as does the proper distance $D$ for the above-mentioned narrowing universe).

The general expressions for the velocity $\chi^{\prime}$ and acceleration $\chi$ "of cosmological ojects in FR $t-\chi$ are easy to find by differentiation (4) or directly from (1)-(3):

$$
\begin{gathered}
\chi^{\prime}=D^{\prime} / R-\left(R^{\prime} / R\right) \chi, \\
\chi^{\prime \prime}=D^{\prime \prime} / R-\left(R^{\prime \prime} / R\right) \chi-2\left(R^{\prime} / R\right)\left[D^{\prime} / R-\left(R^{\prime} / R\right) \chi\right]
\end{gathered}
$$

For planet-like objects (i.e. with $D^{\prime}=0, D^{\prime \prime}=0$ ) from (5)-(6) it is easy to obtain expressions for the velocity $\chi^{\prime}$ and acceleration $\chi^{\prime \prime}$ in FR $t-\chi$.

$$
\chi^{\prime}=-\left(R^{\prime} / R\right) \chi=-H \chi
$$

and

$$
\chi^{\prime \prime}=-\left(R^{\prime \prime} / R\right) \chi+2\left(R^{\prime} / R\right)^{2} \chi=H^{2} \chi
$$

The formula for the velocity $\chi^{\prime}$ is interesting in that it is an analogue of the Hubble law, but in the space $\chi$, not $D$, and with negative $H$. The latter means that 
the object under consideration does not expand, but, on the contrary, narrows in FR $t-\chi$. In order to describe the motion of such an object along the $\chi$ axis, it is convenient to use the distance $f=1-\chi$, measured along the same $\chi$ axis, but in the direction of motion, i.e. from $\chi=1$ to $\chi=0$. Then

$$
\chi^{\prime}=-H f=-H(1-\chi)
$$

The formula (6a) expresses the acceleration $\chi^{\prime \prime}$ through the acceleration $R^{\prime \prime}$ and the speed $R^{\prime}$ of the expansion of the universe. Let's pay attention to the fact that the two terms of the final acceleration $\chi$ "have not only opposite signs, but also completely different status. The real existence of the expansion of the universe with the speed $R^{\prime}$ does not raise the slightest doubt. And the sign of this term is correct, it is it that provides the necessary decrease in time in the modulus of negative velocity (5a). As for the first term in (6a): its existence, of course, cannot be considered firmly established, even despite the results of observations of Sn1a, since other explanations of these results are also possible.

For planets, in accordance to expression (3), it is true:

$$
D^{\prime \prime}=\left(R^{\prime \prime} \chi+R^{\prime} \chi^{\prime}\right)+\left(R^{\prime} \chi^{\prime}+R \chi^{\prime \prime}\right)=\left(v_{\text {reg }}\right)^{\prime}+\left(v_{p e c}\right)^{\prime}=0
$$

Therefore, according to (6), (7) for $R^{\prime \prime}=0$, we have:

$$
\chi^{\prime \prime}=2\left(R^{\prime} / R\right)^{2} f=2 H^{2}(1-\chi)
$$

and, at $R^{\prime \prime}=R H^{2} \chi$

$$
\chi^{\prime \prime}=\left(R^{\prime} / R\right)^{2} f=H^{2}(1-\chi)
$$

Thus, we finally come to the following conclusion: the existence of the acceleration $R^{\prime \prime}$ of the expansion of the universe is not a prerequisite for the occurrence of accelerations $\chi^{\prime \prime}$ acting on objects moving in the FR $t-\chi$, and, moreover, does not have any fundamental effect (sign change) to the final result.

Now let us compare the cosmological accelerations of Hubble and planetary objects. As follows from expression (3), for Hubble objects

$$
D^{\prime \prime}=\chi^{\prime \prime} D=H^{2} R \chi_{i}
$$

But the authors of [2] assume that for all cosmological objects with an increase in $\chi$, the acceleration increases, reaching a maximum of $H^{2} R$ at $\chi=1$. As a result, they obtained the theoretical value of the cosmological acceleration for the Pioneers, which is very far from the observed value.

But what is the difference in the behavior of Hubble and planetary objects? Of course, we are able to compare the behavior of objects only in the same FRs, specifically, their proper FRs, i.e. in FR $t-D$ for Hubble and in FR $t-\chi$ for planets. In this case, the main difference immediately becomes obvious from which all the others should follow. This, of course, is the difference in the signs of the velocities: unconditionally positive for the Hubble velocity $D^{\prime}$ (our Universe is expanding). And, as it turns out, negative value for the proper speed of the planets. What follows from this? That the proper distance $D$ of any Hubble object can only increase over time, i.e. change from smallest to largest. That is why the 
value of the cosmological acceleration $D^{\prime \prime}$ in formula (7) for Hubble objects grows. In contrast, the proper distance $\chi$ can change over time only from larger to smaller. Thus, the projection $\chi$ of the representing point of any object with $D$ $=D_{i}$ in the FR $t-\chi$ necessarily moves along the $\chi$ axis, and in a completely definite way in the direction from the point $\chi=1$ (larger) to the point $\chi=0$ (smaller). Therefore, formula (7) is inapplicable to the motion of all planet-like objects, such as stars in galaxies and galaxies in clusters. The fact is that the law of motion (1) and, consequently, the formula (7) obtained on its basis are valid only for the expanding universe, i.e. for a set of cosmological objects with their own distances increasing over time. Planetary objects do not belong to such. On the contrary, as follows from the law of motion (4), their own distances $\chi$ decrease with time. For them, the point with $\chi=1$ is always the starting point of movement. In the light of the above, we have the only opportunity to measure the distance along the $\chi$ axis: in the direction from the point $\chi=1$ to the point $\chi=0$. Therefore, instead of (8), we must write:

$$
D_{i}^{\prime \prime}=H^{2} R f=H^{2} R(1-\chi)=H^{2} R\left(1-D_{i} / R\right)
$$

Here $f=1-\chi$ is the distance measured along the $\chi$ axis in the direction from $\chi$ $=1$ to $\chi=0$.

Let us estimate what happens to the cosmological acceleration of planet-like objects in the transition from (8) to (9), when the factor $\chi$ in (8) is replaced by the factor $f=(1-\chi)$ in $(9)$. Since the factor $\chi$ is usually extremely small, $D_{i}^{\prime \prime}$ in this case increases by many orders of magnitude. Thus, in [2], using formula (8), the cosmological acceleration of the Pioneers was calculated (at that moment, the Pioneers' proper distance was $40 \mathrm{AU}$ ), equal to $2 \times 10^{-23} \mathrm{~ms}^{-2}$, which is 13 orders of magnitude different from the observed value $2 \times 10^{-10} \mathrm{~ms}^{-2}$. The use of formula (9) instead of (8) gives a calculated value that coincides with the observed one. And for the largest known gravitationally bound object (a cluster of galaxies with a diameter of 30 million light years) we have $f=1-\chi=1-10^{-3}$. Thus, the value $f=(1-\chi)$ that determines the value of $D_{i}^{\prime \prime}$ in (9) is practically equal to 1 for all existing (or at least known to us) gravitationally coupled systems in the visible universe, from planets $\left(\chi=10^{-15}\right)$ and to the largest galaxy clusters $\left(\chi=10^{-3}\right)$. Therefore, practically for all such objects, $D_{i}^{\prime \prime}=H^{2} R$.

Well, it seems that we got a consistent picture, in which the final acceleration $\chi$ "of the planet (necessarily positive in the expanding universe for any values of $R^{\prime \prime}$ ) causes a decrease in the modulus of its negative velocity from (5a) over time.

This acceleration affects the components of all local gravitationally coupled systems. Such as Pioneers, planets, stars in galaxies and galaxies in clusters of galaxies. It turns out that this acceleration is practically independent of $\chi$ and is equal to the fundamental value $R H^{2}=C^{2} / R$.

\section{Conclusions}

It seems that the results obtained are quite enough to explain both the observed anomalous Pioneer effect and the flattened shape of the rotation curves of galax- 
ies. Moreover, a persistent feeling arises that within the framework of the outlined approach, over time, it will be possible to explain most of the effects and phenomena currently associated with the hypothesis of dark matter.

Indeed, what is actually happening? As a result of the local process of stopping the expansion of space, in addition to the "seed" gravitational field, a field of cosmological inertial fields arises. The two fields are summed up, forming a more intense final field in a vast area of space, which, due to the principle of equivalence, is perceived as purely gravitational. Thus, around each center of gravitational attraction (galaxies, clusters of galaxies), in addition to its own gravitational field, a much more extended and much more intense final field is formed at large distances. It is very likely that it is the totality of these fields that is perceived as the gravitational field of the totality of visible matter and dark matter.

The existence of the cosmological inertial field in the expanding universe and its effect on gravitationally bound systems can explain not only the shape of the observed rotation curves, but also numerous other phenomena today explained by the existence of dark matter [3]; such as the results of gravitational microlensing, the formation of galaxies in an obscenely short period of time, or even the well-known picture of the collision of two clusters of galaxies MACSJ0025, in which these clusters, together with "dark matter" (and in our case, with cosmological inertial fields) freely fly through each other, while clouds of intergalactic gas, together with their cosmological inertial fields, are decelerated by collisions. The fact is that particles of hot intergalactic gas moving chaotically, especially in the process of cloud collisions, can have both positive and negative acceleration. Therefore, the total acceleration of all particles, i.e. the field of inertia of an intergalactic gas cloud can turn out to be arbitrarily small, in contrast to the fields of inertia of galaxies in clusters that have flown through each other. Thus, inertial fields can have additional degrees of freedom and thus provide a significant variety in the behavior of various galaxies and their clusters.

It seems that it is the cosmological inertial field that we are discussing (can we call it the inertial field of the universe, the Mach field?). That may turn out to be the physical embodiment of "dark matter".

And in conclusion, let us note that we obtained all the above results exclusively within the framework of the general theory of relativity, without invoking any new entities or hypotheses, such as hitherto unknown particles that make up dark matter, or a modification of Newton's laws. It is the application of general relativity to two classes of cosmological objects (Hubble and planet-like) that made it possible to calculate the cosmological accelerations of planet-like objects in this work.

P.S.: In this work, we were interested in cosmological accelerations only for vanishingly small values of $\chi$, which is quite sufficient when considering local systems. But what happens across the entire $\chi$ range? It turns out in [4], which while at small $\chi$ the cosmological attraction force considered in this article pre- 
vails, at large $\chi$ the repulsive force becomes the main one. So, for a particle with a peculiar velocity, which has the same modulus as in (5a), but opposite sign (direction) $\chi^{\prime \prime}=-4 H^{2} \chi$.

So, what happens? Until now, by default, it is assumed that the same cosmological acceleration $\chi^{\prime \prime}=H^{2} \chi$ acts on each particle, regardless of the direction (sign) of its peculiar velocity. Summing up the result for all particles, we got the final positive acceleration, which could explain the observed cosmological acceleration of the global expansion of the universe. But now the picture is getting more complicated. A braking force acts on the para particles (the $v_{r e g}$ and $v_{p e c}$ directions are antiparallel), which slows down their dispersal, i.e. slowing down the expansion of the universe. And on the ortho particles (the directions $V_{r e g}$ and $V_{p e c}$ are parallel) twice as intense accelerating field, "accelerating" the expansion of the universe. But besides, as was shown in [4], different factors are involved in the expressions for the ortho $(\chi)$ and pair $(-\chi)$ accelerations. Ortho-force is proportional to the factor $f=(1-\chi)$, and para-force is proportional to the factor $\chi$. Therefore, at small $\chi$ we have a net effect that slows down the expansion of the universe, and at large $\chi$ an accelerating one. Of course, the above reasoning is very arbitrary. The point is that the decelerating and accelerating forces are applied to different objects that are in no way connected with each other, except for the general location $\chi$. But in this case, the center of gravity of the cloud (set) of such particles will shift in a completely definite (by the value of $\chi$ ) direction. It is believed [5] that in the real universe, the acceleration of expansion "turned on" after several billion years after the Big Bang.

Summing up the orto/para effects, we obtain for two particles, the final result of the type $\chi_{1}^{\prime \prime}+\chi_{2}^{\prime \prime}=2 H^{2} f-4 H^{2} \chi$, i.e. the sign (direction) of the final acceleration now really depends on the value of $\chi$. So, at $\chi=0.1$ we get $f=0.9$ and the final "braking acceleration" $\chi_{1}^{\prime \prime}+\chi_{2}^{\prime \prime}=1.4 H^{2} \chi$. And at $\chi=0.9$ we have $\chi_{1}^{\prime \prime}+\chi_{2}^{\prime \prime}=-3.4 H^{2} \chi$, i.e. real acceleration of expansion. The braking and accelerating forces balance each other at $\chi=0.33$, i.e. at $f=0.67$. This corresponds to $13.8 \times 0.67=9.2$ billion since the Big Bang, which is in good agreement with the data from [5].

It is also worth noting that cosmological acceleration in the universe acts as a typical positive feedback loop. In the sense, the system pushes each particle in the direction of its motion (of course, in FR $t-\chi$ ).

\section{Conflicts of Interest}

The author declares no conflicts of interest regarding the publication of this paper.

\section{References}

[1] Wallander, S.V. (1978) Lectures on Hydromechanics. Leningrad State University Publishing House, Leningrad.

[2] Carrera, M. and Giulini, D. (2010) Reviews of Modern Physics, 82, 169. https://www.ugr.es/ zink/cosmo/GiuliniRMP.pdf 
https://doi.org/10.1103/RevModPhys.82.169

[3] Zasov, A.V., Saburova, A.S., Khoperskov, A.V. and Khoperskov, S.A. (2017) UFN, 187, 3-44. https://doi.org/10.3367/UFNr.2016.03.037751

[4] Sitnikov, L.S. (2020) On Cosmological Inertial Fields in the Expanding Universe. https://drive.google.com/open?id=1MstLNxIuqcP34TMLiWnqQKgjz0kShUUC

[5] Ask Ethan \# 52: How Long Has It Been Since the Expansion of the Universe Began to Accelerate? https://habr.com/ru/post/395005/ 